eine detaillierte Mitteilung der bisher bekannten Fälle gleichartiger Geschwülste. v. H.

12) de Quervain, F., De l'origine des néoplasmes malins. La Semaine médicale, Année 23, 1903, No. 39.

Zusammenfassende Besprechung über den Ursprung des Krebses an Hand der neueren Literatur. Model (Strassburg i. E.).

13) von Brunn, Ueber den primären Krebs der Extremitäten. Beiträge zur klinischen Chirurgie, Bd.. 37.

Den von $R$ udolf Volkmann 1890 aus der Literatur zusammengestellten 223 Fällen von primärem Extremitätencarcinom fügt v. B r u n 1 145 hinzu. Diese 368 Fälle teilt v. B. nach Volkmanns Vorgang. hinsichtlich der Aetiologie ein in

1) aus Narben und Fisteln und chronischen Entzündungen entstandene: 227 (in 80 Proz. der Fälle bestand die prädisponierende Erkrankung länger als 10 Jahre),

2) aus Warzen (hauptsächlich Keratosen): 46 ,

3) anscheinend aus heiler Haut entstandene: 48,

4) ohne bestimmte Angabe: 47.

In der ersten Gruppe sind relativ am stärksten vertreten die Knochenfisteln (nach Ostitis oder komplizierten Frakturen), dann die chronischen Hautulcera besonders am Unterschenkel und die Brandnarben; damit hauptsächlich glaubt v. B. das in seiner Zusanmenstellung der gesamten Fälle auffallende Ueberwiegen der Männer ïber die Frauen (70 Proz. : 30 Proz.) erklären zu können. In merkwürdigem Widerspruch dazu steht $\mathrm{Heimann}$ vor von Brunn angeführter Berechnung, der in den Zählkartenmaterial des Preussischen statistischen Bureaus unter 207 Fällen beide Geschlechter genau gleichstark betroffen fand.

In v. B.s Zusammenstellung waren 70 Proz. der Kranken zwischen 40 und 70 Jahren alt.

Von 246 verwertbaren Operierten starben 31 infolge der Operation; von den übrigen 215 sind 57 für den definitiven Ausgang nicht verwertbar.

Von dem Rest von 158 Operierten blieben länger als 2 Jahre geheilt 73, bekamen Rezidive 85 (davon 30 nach Amputation).

Drüsenmetastasen sind nicht selten; freilich ist die makroskopische Diagnose unsicher, weil auch sehr vergrösserte Drüsen mikroskopisch von Carcinom frei sein können und umgekehrt. v. B. empfiehlt darum auch für die etwas vorgeschrittenen Extremitätencarcinome die grundsätzliche Exstirpation geschwollener regionärer Drüsen.

\title{
Milner (Berlin).
}

14) Cancer research in Australia. The Empire Review, Vol. 7 , März 1904, No. 38, p. 117.

Dr. Cooke-Adams veröffentlicht in dieser nicht der medizinischen Wissenschaft gewidmeten Zeitschrift eine Uebersicht der 